\title{
Highlights on rare charged Kaon decays
}

\author{
M. Raggi a
}

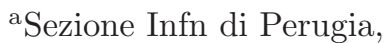

via Pascoli, 06100 Perugia, Italia

Two new results of NA48/2 in the charged K rare decays, based on a fraction of the 2003 data only, will be reported. The first measurement of the DE and INT terms of the decay $K^{ \pm} \rightarrow \pi^{ \pm} \pi^{0} \gamma$, based on $124 \mathrm{~K}$ events, has been performed in the $T_{\pi}^{*}$ region $0<T_{\pi}^{*}<80 \mathrm{MeV}$ :

$$
\begin{aligned}
& \operatorname{Frac}(D E)_{0<T_{\pi}^{*}<80 \mathrm{MeV}}=\left(3.35 \pm 0.35_{\text {sta }} \pm 0.25_{\text {sys }}\right) \% \\
& \operatorname{Frac}(I N T)_{0<T_{\pi}^{*}<80 \mathrm{MeV}}=\left(-2.67 \pm 0.81_{\text {sta }} \pm 0.73_{\text {sys }}\right) \%
\end{aligned}
$$

The best measurement of the $K_{e 4}^{00}$ branching ratio has been performed leading to the result:

$$
B R\left(K_{e 4}^{00}\right)=\left(2.587 \pm 0.026_{\text {sta }} \pm 0.019_{\text {sys }} \pm 0.029_{\text {ext }}\right) \cdot 10^{-5}
$$

\section{NA48/2 experiment}

The NA48/2 detector is essentially based on the existing NA48[1] one with a new beam line designed in order to provide simultaneous $K^{+}$and $K^{-}$beams overlapping in the decay region. The beam, produced using primary $400 \mathrm{GeV}$ protons from the SPS accelerator at CERN, has narrow momentum band $\left(P_{K}=\right.$ $60 \pm 3 \mathrm{GeV})$. The beam line has been equipped with a new detector KABES (KAon BEam Spectrometer)[2] able to measure the Kaon momentum and direction with high precision. The NA48/2 experiment data taking period extends in the summer of 2003 and 2004. What will be discussed in this paper has been obtained using half of the 2003 data only.

\section{Introduction to $K^{ \pm} \rightarrow \pi^{ \pm} \pi^{0} \gamma$ decay}

Nowadays the Chiral Perturbation Theory (ChPT) is one of the most reliable tool to describe low energy QCD dynamics. In this theoretical framework the chiral anomaly plays a crucial role, and has been therefore object of intense studies. Unfortunately precise experimental tests of the chiral anomaly are at the moment very few. Has been noticed that the genuine manifestation of the chiral anomaly in non-leptonic decays, is restricted to the radiative decay of $K^{ \pm} \rightarrow \pi^{ \pm} \pi^{0} \gamma$ in the charged kaon sector.

Two different processes can be responsible for the origin of the gamma. It can be either produced by a final state radiation of the $\pi^{ \pm}$, Inner Bremsstrahlung (IB), or in the decay itself, Direct Emission (DE). Although, due to the dominant IB, the DE component is very difficult to observe it can be isolated kinematically. The DE component consists of magnetic and electric transitions. While the magnetic part can be evaluated using the Wess-Zumino-Witten functional, there is no definite prediction from ChPT on the electric transition, whose amplitude depends on undetermined constants. The electric contribution is extremely interesting since it interferes (INT) with the IB amplitude therefore it may be distinguished from the magnetic, which does not.

In the $K^{ \pm} \rightarrow \pi^{ \pm} \pi^{0} \gamma$ decay IB, INT, and DE can be distinguished kinematically using 


\begin{tabular}{lccc}
\hline exp. & year & \# events & $B R(D E) \cdot 10^{-6}$ \\
\hline E787 [5] & 2000 & $20 \mathrm{~K}$ & $4.7 \pm 0.8 \pm 0.3$ \\
E470 [6] & 2003 & $4.5 \mathrm{~K}$ & $3.2 \pm 1.3 \pm 1.0$ \\
E787 [7] & 2005 & $20 \mathrm{~K}$ & $3.5 \pm 0.6 \pm 0.35$ \\
E470 [8] & 2005 & $10 \mathrm{~K}$ & $3.8 \pm 0.8 \pm 0.7$ \\
\hline
\end{tabular}

Table 1

The $K^{ \pm} \rightarrow \pi^{ \pm} \pi^{0} \gamma$ experimental results

the variable $\mathrm{W}$ which is defined as follows[3]:

$$
W^{2}=\frac{\left(P_{K}^{*} \cdot P_{\gamma}^{*}\right)\left(P_{\pi}^{*} \cdot P_{\gamma}^{*}\right)}{\left(m_{K} m_{\pi}\right)^{2}}
$$

with $P_{x}^{*}$ the 4 -momentum of the x particle and $\gamma$ the radiative one. The decay rate depends only on $T_{\pi}^{*}$, energy of the pion in the Kaon rest frame, and $\mathrm{W}$. Integrating over $T_{\pi}^{*}$ an expression that separates the different contributions into terms with different powers of $\mathrm{W}$ can be obtained:

$$
\begin{aligned}
\frac{d \Gamma^{ \pm}}{d W} \simeq & \left(\frac{d \Gamma^{ \pm}}{d W}\right)_{I B}[1+ \\
& +2\left(\frac{m_{\pi}}{m_{K}}\right)^{2} W^{2}|E| \cos \left(\left(\delta_{1}-\delta_{0}\right) \pm \phi\right) \\
& \left.+\left(\frac{m_{\pi}}{m_{K}}\right)^{4} W^{4}\left(|E|^{2}+|M|^{2}\right)\right]
\end{aligned}
$$

The three terms represent IB, INT and DE contribution respectively.

\subsection{Present experimental knowledge}

The IB component has been measured since the seventies by Abrams et al. [4] achieving a good agreement with solid QED theoretical predictions. The experimental measurement of the fractions of DE and INT is affected by very dangerous BG sources, such as $K^{ \pm} \rightarrow$ $\pi^{ \pm} \pi^{0}$ and $K^{ \pm} \rightarrow \pi^{ \pm} \pi^{0} \pi^{0}$ decays, suppressed in a kinematically $\mathrm{BG}$ free region, $T_{\pi}^{*}$ in the range $55-90 \mathrm{MeV}$. Moreover a good measurement requires a very good reconstruction of both charged and neutral particles 4-momenta. The present experimental knowledge on the $K^{ \pm} \rightarrow \pi^{ \pm} \pi^{0} \gamma$ decay is summarized in tab.1.

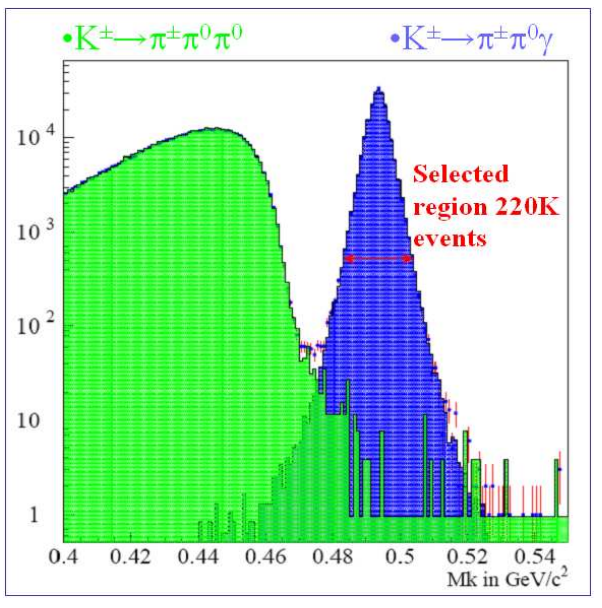

Figure 1. Data-mc comparison of $M_{K}$ spectrum.

All the results have been obtained in the hypothesis of vanishing interference and in the $T_{\pi}^{*}$ region $55-90 \mathrm{MeV}$.

\subsection{Selection and backgrounds}

The main BG sources are $K^{ \pm} \rightarrow \pi^{ \pm} \pi^{0}$ and $K^{ \pm} \rightarrow \pi^{ \pm} \pi^{0} \pi^{0}$. The first decay needs an accidental photon or an hadronic extra cluster to mimic the signal final state, while the second a lost or 2 fused gamma. The selection aims to suppress the contribution of them both to less than $1 \%$ of the DE component. The rejection of $K^{ \pm} \rightarrow \pi^{ \pm} \pi^{0}$ relays on the $T_{\pi}^{*}$ cut. The request $T_{\pi}^{*}$ lower than $80 \mathrm{MeV}$ allows to reject $K^{ \pm} \rightarrow \pi^{ \pm} \pi^{0}$ and a part of the IB spectrum of $K^{ \pm} \rightarrow \pi^{ \pm} \pi^{0} \gamma$ only, including region 0-55 MeV very rich of DE and INT events. The upper cut at $80 \mathrm{MeV}$ is due to trigger reasons. To suppress $K^{ \pm} \rightarrow \pi^{ \pm} \pi^{0} \pi^{0} \mathrm{BG}$ the very good kaon mass resolution, $(2.2 \mathrm{MeV})$, and the identification of fused gamma events constraints have been used. In fig. 1 the data kaon mass spectrum is compared with the sum of $K^{ \pm} \rightarrow \pi^{ \pm} \pi^{0} \gamma$ and $K^{ \pm} \rightarrow \pi^{ \pm} \pi^{0} \pi^{0} \mathrm{MC}$.

A very important issue in the measurement 


\begin{tabular}{lcc}
\hline Effect & syst. DE & syst. INT \\
\hline Energy scale & 0.09 & -0.21 \\
LKr non linearity & $<0.05$ & $<0.05$ \\
$\gamma$ misidentification & - & \pm 0.2 \\
Fitting procedure & 0.02 & 0.019 \\
Resolution difference & $<0.05$ & $<0.1$ \\
LVL1 trigger & \pm 0.17 & \pm 0.43 \\
LVL2 Trigger & \pm 0.17 & \pm 0.52 \\
BG contributions & $<0.05$ & $<0.05$ \\
\hline TOTAL & \pm 0.25 & \pm 0.73 \\
\hline
\end{tabular}

Table 2

Systematic checks results

of DE and INT is to identify the radiative $\gamma$ among the 3 available. A dedicated set of cuts, based on the agreement of the vertex evaluated using the pion and kaon tracks, and the

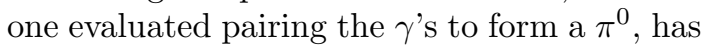
been implemented. Using this cuts a misidentification probabilities, computed using $\mathrm{MC}$ simulation, of the order of the permille for all the components has been achieved. A total of $\sim 220000$ candidate events survived all selection cuts in the region of $T_{\pi}^{*} 0-80 \mathrm{MeV}$.

\subsection{Measurement technique}

The extraction of the fractions of DE and INT relies on the fact that different components show quite different $\mathrm{W}$ distributions. An extended maximum likelihood technique, assigning weights to $\mathrm{MC} \mathrm{W}$ distributions of the 3 components to reproduce data spectrum, has been used to get the fractions.

Many checks to verify the result were made which are summarized in table 2 . They include checks on the $\gamma$ energies reconstructed by the calorimeter, on trigger efficiencies and on BG contribution. The systematic uncertainties are dominated by the trigger.

The fit has been performed in the interval $0.2-0.9$ in the $W$ variable using only $124 \mathrm{~K}$ events from the total sample. After correcting for different acceptances we get, in the region $0<T_{\pi}^{*}<80 \mathrm{MeV}$, the following values for the

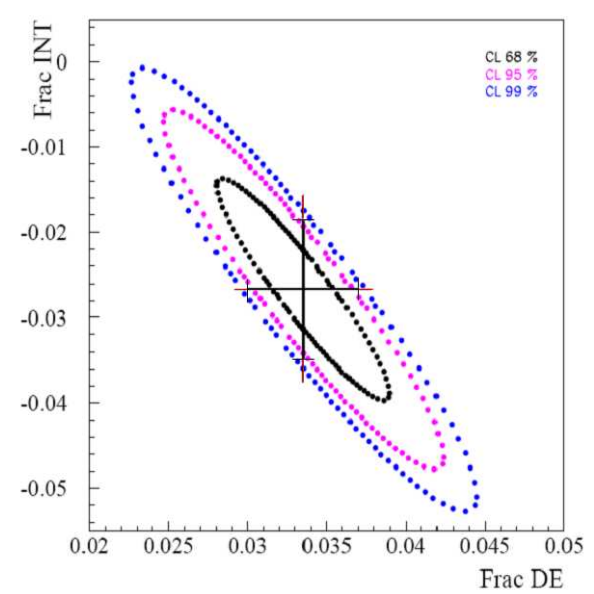

Figure 2. Contour plot for DE and INT components

fractions of DE and INT with respect to IB:

$$
\begin{array}{r}
\operatorname{Frac}(D E)=\left(3.35 \pm 0.35_{\text {sta }} \pm 0.25_{\text {sys }}\right) \% \\
\operatorname{Frac}(I N T)=\left(-2.67 \pm 0.81_{\text {sta }} \pm 0.73_{\text {sys }}\right) \%
\end{array}
$$

This is the first measurement of a non vanishing interference term in the $K^{ \pm} \rightarrow \pi^{ \pm} \pi^{0} \gamma$ channel. The contour plot in figure 2 shows the very high correlation of the two contributions.

\section{The Ke4 neutral decay}

The $K \rightarrow \pi \pi e \nu$ decays are a very interesting field to study many properties of QCD dynamics. In fact the decay form factors provide useful constraints on the parameters of the ChPT Lagrangian. They are also clean sources of low energy of $\pi-\pi$ pairs, from which one can extract the scattering lengths of significance for models of hadron dynamics. In the charged kaon sector there are two kind of Ke4 decays, $K_{e 4}^{ \pm}, K_{e 4}^{00}$. NA48/2 plans to study in detail both of them with very high statistic data samples. Since Ke4 decays are theoretically related to each other by isospin arguments, experimental studies for all channels 
will provide a complete understanding of $\mathrm{Ke} 4$ physics. Among the Ke4 decays, the $K_{e 4}^{00}$ is the simplest because the decay kinematics can be described by only one form factor, due to the presence of two identical $\pi^{0}$ 's in the final state.

Up to now the best measurement of the properties of the $K_{e 4}^{00}$, based on 216 events, has been performed by the E470 at KEK[9].

\subsection{BR and form factor measurement}

The $K_{e 4}^{00}$ is affected by two main BG sources $K^{ \pm} \rightarrow \pi^{ \pm} \pi^{0} \pi^{0}$ and $K^{ \pm} \rightarrow \pi^{0} e^{ \pm} \nu(\gamma)$. In the first case the pion has to be misidentified as an electron, while for the radiative Ke3 another accidental gamma is required, to reproduce the signal final state. The selection uses the compatibility of 2 the neutral vertices, evaluated using $\gamma$ pairs, to assign two $\gamma \mathrm{s}$ to each of the two $\pi^{0}$, and assume the Kaon momentum to be $60 \mathrm{GeV} / \mathrm{c}$ to compute the transverse momentum of the neutrino, and then the kaon mass. At the end of the selection, on 2003 data only, a sample of 9642 candidate events has been identified with an estimated background of $260 \pm 96$ events, coming form $K^{ \pm} \rightarrow \pi^{ \pm} \pi^{0} \pi^{0}$, and $16 \pm 2$ from Ke3 $\gamma$. Using the $K^{ \pm} \rightarrow \pi^{ \pm} \pi^{0} \pi^{0}$ decay as normalization channel to compute the total kaon flux, the $B R\left(K_{e 4}^{00}\right)$ has been evaluated to be:

$$
f_{s}^{\prime \prime} / f_{s}=-0.040 \pm 0.034_{\text {sta }} \pm 0.020_{\text {sys }}
$$

Those values are consistent with the ones measured by NA48/2, with higher statistical sensitivity, in the $K_{e 4}^{ \pm}$channel.

\section{REFERENCES}

1. V. Fanti et al. [NA48 Collaboration], Phys. Lett. B 465, 335 (1999)

2. B. Peyaud, Nucl. Instrum. Meth. A 535, 247 (2004).

3. J. D. Good, PHYS. REV. 113, 352 (1959).

4. R. J. Abrams et al., Phys. Rev. Lett. 29, 1118 (1972).

5. S. C. Adler et al. [E787 Collaboration], Phys. Rev. Lett. 85, 4856 (2000)

6. M. A. Aliev et al. [E470 Collaboration], Phys. Lett. B 554, 7 (2003)

7. T. Tsunemi, "New Results on $K^{+} \rightarrow$ $\pi^{+} \pi^{0} \gamma$ from E787", talk given at Kaon 2005 Chicago, June 2005.

8. M. A. Aliev et al. [E470 Collaboration], [arXiv:hep-ex/0511060 v1], 2005

9. S. Shimizu et al. [E470 Collaboration], Phys. Rev. D 70, 037101 (2004)

$B R\left(K_{e 4}^{00}\right)=\left(2.587 \pm 0.026_{\text {sta }} \pm 0.019_{\text {sys }} \pm 0.029_{\text {ext }}\right) \cdot 10^{-5}$

Due to the presence of two identical $\pi^{0}$ 's in the final state in this Ke4 channel there is only one form factor $\mathrm{F}$ :

$$
F=f_{s}+f_{s}^{\prime} q^{2}+f_{s}^{\prime \prime} q^{4}+f_{e}\left(S_{e} / 4 m_{\pi}^{2}\right)+\ldots
$$

To enhance the sensitivity to the form factors the fit has been performed using both 2003 and 2004 data $(\sim 38 \mathrm{~K}$ events$)$. In the first fit attempt unfortunately no sensitivity to $f_{e}$ has been reached and then a second fit has been performed assuming $f_{e}=0$. Under this assumption we get:

$$
f_{s}^{\prime} / f_{s}=0.129 \pm 0.036_{\text {sta }} \pm 0.020_{\text {sys }}
$$

